\title{
Constraining deflagration models of Type Ia supernovae through intermediate-mass elements
}

\author{
D. García-Senz ${ }^{1,2}$, E. Bravo ${ }^{1,2}$, R.M. Cabezón ${ }^{1}$ and S.E. Woosley ${ }^{3}$
}

\begin{abstract}
The physical structure of a nuclear flame is a basic ingredient of the theory of Type Ia supernovae (SNIa). Assuming an exponential density reduction with several characteristic times we have followed the evolution of a planar nuclear flame in an expanding background from an initial density $6.6 \times 10^{7} \mathrm{~g} . \mathrm{cm}^{-3}$ down to $2 \times 10^{6} \mathrm{~g} . \mathrm{cm}^{-3}$. The total amount of synthesized intermediate-mass elements (IME), from silicon to calcium, was monitored during the calculation. We have made use of the computed mass fractions, $\mathrm{X}_{I M E}$, of these elements to give an estimation of the total amount of IME synthesized during the deflagration of a massive white dwarf. Using $\mathrm{X}_{I M E}$ and adopting the usual hypothesis that turbulence decouples the effective burning velocity from the laminar flame speed, so that the relevant flame speed is actually the turbulent speed on the integral length-scale, we have built a simple geometrical approach to model the region where IME are thought to be produced. It turns out that a healthy production of IME involves the combination of not too short expansion times, $\tau_{c} \geq 0.2 \mathrm{~s}$, and high turbulent intensities. According to our results it could be difficult to produce much more than $0.2 \mathrm{M}_{\odot}$ of intermediate-mass elements within the deflagrative paradigma. The calculations also suggest that the mass of IME scales with the mass of Fe-peak elements, making it difficult to conciliate energetic explosions with low ejected nickel masses, as in the well observed SN1991bg or in SN1998de. Thus a large production of Si-peak elements, especially in combination with a low or a moderate production of iron, could be better addressed by either the delayed detonation route in standard Chandrasekhar-mass models or, perhaps, by the off-center helium detonation in the sub Chandrasekhar-mass scenario.
\end{abstract}

Subject headings: supernova: general, explosive nucleosynthesis

\section{Introduction}

A distinctive feature of SNIa spectra near maximum light is the presence of intermediate-mass elements from Si to Ca moving at velocities 10000-16000 $\mathrm{km} . \mathrm{s}^{-1}$. In particular the presence or not of the SiII absorption feature at $6150 \AA$ is basic to identify a Type I supernovae as SNIa. According to observations about $0.2-0.4 \mathrm{M}_{\odot}$ of IME have to be synthesized during the explosion. An amount of IME larger than $0.2 \mathrm{M}_{\odot}$ is also needed to explain the observed abun-

\footnotetext{
${ }^{1}$ Departament de Física i Enginyeria Nuclear, UPC, Jordi Girona 3, Mòdul B5, 08034 Barcelona, Spain; domingo.garcia@upc.edu; eduardo.bravo@upc.edu; ruben.cabezon@upc.edu

${ }^{2}$ Institut d'Estudis Espacials de Catalunya, Barcelona, Spain

${ }^{3}$ Department of Astronomy and Astrophysics, 477 Clark Kerr Hall, University of California, Santa Cruz, CA 95064; woosley@ucolick.org
}

dances of these elements in the solar neighborhood (Iwamoto et al. 1999). Therefore, any attempt to build a satisfactory theoretical model of these explosions has to give the right amount of intermediate-mass elements.

In this respect the underproduction of intermediatemass elements found in current pure deflagration models of Type Ia supernovae is a long standing problem of theoretical modelization of these explosions. On fundamental basis we know that these elements have to be synthesized below densities $\rho \simeq 5 \times 10^{7} \mathrm{~g} . \mathrm{cm}^{-3}$ in an expanding medium. Nevertheless the resolution achieved by the best multidimensional models carried out to date is far from adequate to solve unambigously this problem. In this work we have taken another route to address this issue. Instead of performing a multidimensional calculation incorporating the unresolved scales as a subgrid we have simulated the evolution 
of an expanding nuclear flame in planar aproximation with very good resolution. We have then used the results to put limits to the amount of IME synthesized in a deflagration. The usefulness of our approximation requires that the synthesis of elements within the mass range $28 \leq \mathrm{A} \leq 40$ takes place, for the most part, during the flamelet regime. In such a regime the flame, although highly wrinkled and distorted, still preserves its identity. Unfortunately the precise value of the minimum density at which the flamelet hypothesis still holds is not known. There are indications that transition from the flamelet to the distributive regime in the combustion diagram could begin around $2 \times 10^{7}$ g.cm ${ }^{-3}$ (Niemeyer et al. 1999; Zingale et al. 2005), and we can take it as a reference.

Keeping that constraint in mind we have proceeded in the following way. First, the detailed structure and evolution of a nuclear flame propagating through an expanding background has been solved for a reasonable set of characteristic expansion times and the nucleosynthetic yields calculated using a simplified $\alpha$ network of 14 nuclei, $\S 2$. With that information we provide in $\S 3$ a rough calculation of the total amount of IME synthesized in a typical deflagration of a white dwarf as a function of the effective surface of heat exchange between ashes and fuel at the densities of interest, the turbulence intensity and the characteristic expansion time. According to our results a subsonic deflagration is able to produce more than $0.2 \mathrm{M}_{\odot}$ of IME although only in a limited region of parameter space. This point is discussed in the chapter devoted to the conclusions, $\S 4$, as well as possibles issues and limitations of our work.

\section{Production of Intermediate-mass elements in an expanding laminar flame}

From the nucleosynthetic point of view IME are the main product of incomplete $\mathrm{Si}$ burning at $T_{9} \leq$ 5 followed by $\alpha$-rich freeze-out until the quenching of nuclear reactions. During the explosion of a massive white dwarf such combustion regime is attained when the density ahead the flame drops below $\simeq 5 \times 10^{7}{\mathrm{~g} . \mathrm{cm}^{-3}}^{-}$in a medium which is rapidly expanding. As density decreases, the laminar velocity of the flame goes down, its width enlarges and the nucleosynthetic products are enriched first in IME and later in ${ }^{24} \mathrm{Mg}$ and ${ }^{16} \mathrm{O}$. Finally the flame becomes a thermal wave and no more nuclear combustion takes place: the flame dies. Even though the thickness of the zone where IME are synthesized in the planar case is already really narrow, around half kilometer (see below), the surface of the flame in a real situation is so distorted by hydrodynamical instabilities that the effective volume might be enough to account for the observed amount of IME. One important consequence is that, once developed, the hydrodynamical instabilities make the effective burning velocity independent of the laminar velocity of the flame $(\S 3)$.

We have devised a numerical experiment to investigate whether or not the adequate amount of IME can be produced by the above mechanism. First of all we have made an estimation of the total amount of chemical species generated by a planar flame moving through a rapidly expanding media. Although in the end the effective turbulent burning velocity will become independent of the laminar value such calculation is worthwhile because: 1) it provides the mass fraction of the isotopes of interest to be used in the next section when turbulence effects are taken into account, 2) It shows the futility of trying the synthesis of IME in a real explosion when the flame only moves at its laminar speed, and 3) knowing the physics of an expanding planar nuclear flame is an interesting topic by itself as it represents a natural extension of the work by Timmes \& Woosley (1992) (TW afterwards). The ouptut of the expanding planar flame calculation gives a fundamental quantity: $\Delta m_{I M E}$, the mass of IME synthesized in a column with $1 \mathrm{~cm}^{2}$ section. For a given amount of burnt material $\Delta m_{\text {burnt }}$ we compute the mass fraction of IME, $\mathrm{X}_{I M E}$ synthesized below a fixed starting density $\rho_{0}$ for different expansion rates. Later on $(\S$ 3) we make use of $\mathrm{X}_{I M E}$ to get an estimation of the total amount of IME ejected during a typical Type Ia supernova event. The quantity $\Delta m_{I M E}$ was calculated by following the propagation of the nuclear flame in planar geometry as explained in Cabezón et al. (2004). Basically the integration scheme uses an $\alpha$-network with 14 nuclei and implicit thermal coupling with the energy equation to follow the combustion under isobaric conditions. The resulting method is robust and stable, allowing to properly handle the rear tail of the flame where most of the elements of interest were synthesized. In addition an exponential reduction in density ahead the flame, with a characteristic time $\tau_{c}$, is imposed. The resulting set of equations are:

$$
\frac{d Y_{i}}{d t}=\sum_{k, l} r_{k l} Y_{k} Y_{l}-\sum_{j} r_{i j} Y_{i} Y_{j}+\sum_{m} \lambda_{m} Y_{m}-\lambda_{i} Y_{i}
$$




$$
\begin{gathered}
\frac{d U}{d t}=\frac{1}{\rho} \frac{\partial}{\partial x}\left(\sigma \frac{\partial T}{\partial x}\right)+\frac{P}{\rho^{2}} \frac{d \rho}{d t}+\dot{\epsilon}_{n u c} \\
\rho\left(x>>x_{\text {flame-front }}\right)=\rho_{0} \exp \left[-t / \tau_{c}\right] \\
P(t)=P_{\text {ext }}(\rho, T)
\end{gathered}
$$

where $r_{i j}$ stands for particle reaction rates, $\lambda_{i}$ is for photodisintegrations and the rest of the symbols have their usual meaning. The thermal conductivity $\sigma$ was taken from Appendix 1 in Khokhlov et al. (1997) and we have taken an uniform initial density of the fuel $\rho_{0}=6.6 \times 10^{7} \mathrm{~g} . \mathrm{cm}^{-3}$. The precise value of $\rho_{0}$ is irrelevant for the results as long as it is larger than the density at which IME begin to be produced. At each step we enforce the temperature of the unburnt material, located far enough from the flame front, to evolve adiabatically. The instantaneous value of T and $\rho$ far ahead the flame sets the value of $\mathrm{P}_{\text {ext }}$ in Equation (4). Across the flame the evolution is governed by heat difussion and binary ${ }^{12} C+{ }^{12} C$ reaction, whereas in the burned zone far from the flame front the incinerated material relaxes to either Fe-peak elements or to IME if density becomes low enough. The integration scheme is a standard lagrangian code with enough mass-shells to resolve the structure of the flame. At the beginning of the calculation a small group of shells were put into nuclear statistical equilibrium at constant pressure. Very soon a steady nuclear flame developed and propagated through the cold fuel (composed by equal parts of carbon and oxygen). As the flame progressed we kept a constant number of mass-shells ahead the flame (around 100) in order to save computer time. Finally the calculation was stopped when the temperature in the burned zone dropped below two billion degrees and all nuclear reactions virtually quenched. At the end of the calculation there were typically between $2 \times 10^{4}$ to $10^{5}$ shells in combustion, depending on the characteristic expansion time $\tau_{c}$. It was necessary to calculate several millions of time steps in order to follow the whole process.

In Figure 1 it is shown a snapshot of the mass fraction profile at $\mathrm{t}=0.1523 \mathrm{~s}$, which is the time at which total IME abundance freezes in our reference model with $\tau_{c}=0.1 \mathrm{~s}$. As we can see ${ }^{28} \mathrm{Si}$ as well as other IME with higher atomic mass were synthesized in a tiny layer, lesser than $0.3 \mathrm{~km}$, which is more than one order of magnitude below the resolution of the best three-dimensional simulations carried out so far.
Moreover because during the explosion the flame is shattered by turbulence that length represents an upper limit of the size of pockets of ashes made of elements belonging to the silicon family. Elements lighter than silicon such as magnesium and neon were produced at lower density in a very narrow region behind the flame edge. In Figure 2 we depict the evolution of the total synthesized mass of elements between ${ }^{28} \mathrm{Si}$ to ${ }^{40} \mathrm{Ca}$ for $\tau_{c}=0.05,0.1,0.2$ and $0.3 \mathrm{~s}$ as a function of the fuel density. From these figures it is clear that for plausible expansion times the IME are synthesized in the density range $2-5 \times 10^{7} \mathrm{~g} . \mathrm{cm}^{-3}$, as it was recognized many years ago. In particular, we see that $95 \%$ of IME were already synthesized at densities higher than $2 \times 10^{7} \mathrm{~g} . \mathrm{cm}^{-3}$. In fact, more than a half of their final mass was produced when the density of the fuel was still above $4 \times 10^{7} \mathrm{~g} . \mathrm{cm}^{-3}$. This is an interesting feature because it suggests that important nuclei for SNIa spectroscopy, such as silicon and calcium, are probably synthesized during the so-called flamelet regime of turbulent combustion, much more easier to handle than the distributive regime. Below $2.5 \times 10^{7} \mathrm{~g} . \mathrm{cm}^{-3}$ we found an increase of direct carbon and oxygen combustion products, neon and magnesium in our network, in a region of size $\simeq 0.05 \mathrm{~km}$. However in a real physical situation the final abundance of elements below silicon might be altered if combustion enters the distributive regime. The velocities of the flame, fitted using the total amount of burnt fuel (see Eq. 7 below), are in reasonable agreement with those obtained by TW at different densities. We obtain slightly lower values because the limited nuclear network used to carry out the calculations. For example, we obtained $\mathrm{v}=0.14$, 0.23 , and $0.33 \mathrm{~km} . \mathrm{s}^{-1}$ at $\rho_{7}=2,3$ and 4 respectively, to be compared to those calculated by $\mathrm{TW}, \mathrm{v}_{T W}=0.19$, $0.32,0.46 \mathrm{~km} . \mathrm{s}^{-1}$ at the same densities. The instantaneous width of the flame was also consistent with TW calculation. The detailed final yields of the 14 nuclei for several characteristic expansion times are shown in Table 1. As we can see, there is a monotonic increase of the final mass of the most abundant species with the expansion time $\tau_{c}$. For $\tau_{c}=0.05 \mathrm{~s}$ we got $2.95 \times 10^{10}{\mathrm{~g} . \mathrm{cm}^{-2}}^{-}$of silicon whereas for $\tau_{c}=0.3 \mathrm{~s}$ the synthesized mass was $1.16 \times 10^{11} \mathrm{~g} . \mathrm{cm}^{-2}$. The same trend is true for the size of the region where these elements are significatively produced: $\simeq 0.15 \mathrm{~km}$ $\left(\tau_{c}=0.05 \mathrm{~s}\right)$ to $\simeq 0.75 \mathrm{~km}\left(\tau_{c}=0.3 \mathrm{~s}\right)$. Even in the last case the width of the IME layer is much smaller than the size of the burned region, typically $2000 \mathrm{~km}$. Thus in the lagrangian one-dimensional picture it can 
be regarded as a very thin layer on top of a volume made basically of Fe-peak elements.

The mass of fuel consumed during the propagation of an expanding planar flame starting at an initial density $\rho_{0}$ can be found analytically because the density dependence of the laminar velocity is known (TW):

$$
v=v_{0}\left(\frac{\rho}{\rho_{0}}\right)^{n}
$$

From our results $v_{0} \simeq 0.6 \mathrm{~km} . \mathrm{s}^{-1}$ at $\rho_{0}=6.6 \times$ $10^{7} \mathrm{~g} . \mathrm{cm}^{-3}$, and $n \simeq 1.2$. Using Equation 3 the mass of burned fuel per unit surface in an elapsed time $\Delta t$ is:

$$
\begin{array}{r}
\Delta m_{\text {burnt }}=\rho_{0} v_{0} \int_{0}^{\Delta t} \exp \left[-\frac{(1+n) t}{\tau_{c}}\right] d t= \\
\rho_{0} v_{0} \frac{\tau_{c}}{(1+n)}\left[1-\exp \left(-\frac{(1+n) \Delta t}{\tau_{c}}\right)\right]
\end{array}
$$

Taking $\Delta t \rightarrow \infty$ in Equation 6 gives a good approximation to the the total burnt mass per unit surface:

$$
\Delta m_{\text {burnt }}=\rho_{0} v_{0} \frac{\tau_{c}}{(1+n)}
$$

Hence the amount of consumed fuel below the starting density $\rho_{0}$ in the expanding background is simply proportional to the product of the laminar velocity and the characteristic expansion time. The laminar velocity of the flame is rather well known within, at most, a factor two uncertainity. The value of the characteristic expansion time $\tau_{c}$ is probably dependent of the particular explosion dynamics. A rough lower limit for $\tau_{c}$ is the characteristic hydrodynamical time scale $\tau_{h}=446 / \sqrt{\rho}=0.08 \mathrm{~s}$ at $\rho=3 \times 10^{7} \mathrm{~g} . \mathrm{cm}^{-3}$ which matches well also the behaviour of a particular deflagrative one-dimensional model (Bravo et al. 1996) as shown in Figure 3. A reasonable range for $\tau_{c}$ is $0.05 s \leq \tau_{c} \leq 0.3 s$ once the flame has reached densities below $6 \times 10^{7} \mathrm{~g} . \mathrm{cm}^{-3}$, although higher values, nearing 0.5 seconds, can not be totally discarded. In Table 2 there is shown the total amount of burned mass as well as the masses of IME and nickel synthesized below $\rho_{0}$ as a function of $\tau_{c}$. As we can see the burnt mass scales linearly with $\tau_{c}$, as suggested by Equation 7. However, the amount of synthesized IME is not proportional to the characteristic expansion time because a longer value of $\tau_{c}$ leads to the formation of more iron as the nuclear system has more room to relax. A simple fitting formulae to the values of $\triangle m_{I M E}$ given in
Table 2 that can be also used to extrapolate $\Delta m_{I M E}$ beyond $0.3 \mathrm{~s}$, until $\tau_{c} \simeq 0.5 \mathrm{~s}$ if necessary, is:

$$
\Delta m_{I M E}=6.5 \times 10^{11} \tau_{c}^{\frac{3}{4}} \text { g. } \mathrm{cm}^{-2}
$$

As mentioned before, the mass of IME given by Equation 8 is independent of the particular value chosen for $\rho_{0}$ in Equation 3 provided it is high enough to resolve the region where intermediate-mass elements are synthesized. However their mass fraction, $\mathrm{X}_{I M E}$, within the burnt material is a function of the starting density $\rho_{0}$ in the exponential expansion. For $\rho_{0}=6.6 \times 10^{7} \mathrm{~g} . \mathrm{cm}^{-3}$ it can be fitted using:

$$
X_{I M E}=0.35 \tau_{c}^{-\frac{1}{4}}
$$

Another remarkable feature shown in Table 2 is that the production of ${ }^{56} \mathrm{Ni}$ is no longer negligible, especially for $\tau_{c} \geq 0.2 \mathrm{~s}$. At the longest expansion time considered here, $\tau_{c}=0.3 \mathrm{~s}$, the amount of ${ }^{56} \mathrm{Ni}$ is practically equal to that of IME.

\section{Consequences for Type Ia Supernova models}

A quantitative estimation of the total amount of IME ejected in a SNIa explosion may be done provided the effective flame surface at densities $\rho \simeq$ $2-5 \times 10^{7}$ g.cm ${ }^{-3}$ is somehow known. However, this is a difficult task that will have to be addressed in the future through high resolution multidimensional simulations. For the time being we give only a qualitative discussion about this point taking advantage of the selfsimilar behaviour of the main physical agents responsible for the corrugation of the flame. There has been shown (Khokhlov 1995; Niemeyer \& Woosley 1997), that a large increase in flame surface can only be attributed to two physical mechanisms: the RayleighTaylor (RT) instability and turbulence. Fortunately, it turns out that both are self-similar phenomena that can be approximately described using three parameters: the maximum and minimum scale-lengths, $\lambda_{\max }, \lambda_{\min }$ and the average fractal dimension D' of the corrugated flame surface. In this approximation the incinerated mass is given by:

$$
M_{\text {burnt }}=4 \pi \bar{r}^{2}\left\langle\frac{\lambda_{\max }}{\lambda_{\min }}\right\rangle^{D^{\prime}-2} \int_{0}^{\Delta t} \rho v_{\text {flame }} d t
$$

where $\bar{r}$ is the average radius of the flame and $v_{\text {flame }}$ stems for its laminar velocity. Even though 
in the supernova the nuclear flame becomes highly distorted and shattered by turbulence and hydrodynamical instabilities the amout of fuel consumed per unit area given by the integral part of Equation 10 should not be very different from $\Delta m_{\text {burnt }}$ given by Equation 7 corresponding to the planar case analyzed in the previous section. However many experiments in combustion chambers as well as numerical simulations dealing with turbulent combustion suggest that $\lambda_{\text {min }}$ is no longer an independent quantity (Peters \& Franke 1989). Its local value self-adapts so that the effective burning rate is set by the integral length $\lambda_{\max }$ and the characteristic velocity, $\mathrm{V}$, at the integral length-scale. Such interesting behaviour relies in the existence of an universal scaling law for velocity spectrum of the type:

$$
\left(\frac{\lambda_{\max }}{\lambda_{\min }}\right)=\left(\frac{V}{v_{\text {flame }}}\right)^{\frac{1}{D^{\prime}-2}}
$$

which for D' $=7 / 3$ becomes the popular Kolmogorov scaling-law for turbulence. Adopting the scaling relationship given by Equation 11, both $\mathrm{M}_{\text {burnt }}$ as well as the mass of the $\mathrm{k}$-isotope, $\mathrm{M}_{k}$ become independent of $v_{\text {flame }}$. The ejected mass of the $\mathrm{k}$-isotope can therefore be calculated using the value of $\mathrm{X}_{k}$ computed in the previous section:

$$
M_{k}=4 \pi \bar{r}^{2} V\left(\int_{0}^{\Delta t_{k}} \rho(t) d t\right) X_{k}
$$

where $\Delta t_{k}$ is the elapsed time until the abundance of the respective nuclei freezes. For example, as stated in $\S 2$ the nuclear combustion is unable to synthesize nuclei beyond magnesium once the density has gone down $\simeq 1.5 \times 10^{7} \mathrm{~g} \cdot \mathrm{cm}^{-3}$. Within our model of exponential density reduction this occurs once $\Delta t_{I M E}=1.49 \tau_{c}$.

Admittedly, the above discussion is a little artificial, but necessary to develop the formalism, because finally neither $\mathrm{v}_{\text {flame }}$ nor D' appears in Equation 12. According to that equation the progression of the flame can be envisaged as a spherical flame front of radius $\bar{r}$ moving not at the laminar speed but at velocity $\mathrm{V}$. As $\mathrm{V}$ is expected to be many orders of magnitude larger than $v_{\text {flame }}$ the synthesized mass of nickel as well as the released nuclear energy are sufficient to account for the supernova light curve and energetics. Nevertheless a variation of the geometrical approach given by Equation 12 could be more fruitful to address the particular problem of the synthesis of IME (Woosley 2001). In this regard it might be more physical to interpret $\bar{r}$ as the average radius of the ashes, made basically of nuclear-statistical equilibrium elements (NSEE). The intermediate-mass elements are then produced in a tiny shell, almost a surface, on top of the huge volume defined by the underlying ashes, over a length given by the product $\mathrm{V} \Delta t_{I M E}$. Unlike the synthesis of NSEE, which as bounded to a volume are reasonably given by Equation 12, surface effects are important to properly model the synthesis of IME. Assuming that the effective surface of NSEE behaves as a fractal in which those scale-lengths below the current thickness of the IME layer itself do not appreciably contribute to $\mathrm{M}_{I M E}$, we can write:

$M_{I M E}=4 \pi \bar{r}^{2}\left(\frac{\bar{r}}{V \Delta t_{I M E}}\right)^{D-2} V\left(\int_{0}^{\Delta t_{I M E}} \rho(t) d t\right) X_{I M E}$

which either for $\mathrm{D}=2$ or for $\left(\mathrm{V} \Delta t_{I M E}\right) \simeq \bar{r}$ reduces to Equation 12. Here, the fractal dimension $D$ is intended to describe the geometrical properties of the NSEE external surface in between the integral length-scale and the radius of the front, and has not to be the same as the dimension $D^{\prime}$ characterizing the front at lower lengthscales. Finally, to compute $\mathrm{M}_{I M E}$ from Equation 13 it is more convenient to use the mass of the ashes made of NSEE, $M_{N S E E}$, instead their average radius $\bar{r}$. Then, solving for the integral, taking $\mathrm{X}_{I M E}$ from Equation (9) and using $\Delta t_{I M E}=1.49 \tau_{c}$ the resulting expression is:

$M_{I M E}\left(M_{\odot}\right)=0.253\left(\frac{M_{N S E E}\left(M_{\odot}\right)}{\bar{\rho}_{7}}\right)^{D / 3} 2.43^{D} V_{8}^{3-D} \tau_{c}^{11 / 4-D}$

where $\bar{\rho}_{7}$ is the average density of the ashes in $10^{7} \mathrm{~g} \cdot \mathrm{cm}^{-3}$ and $\mathrm{V}_{8}$ the average velocity of the effective flame velocity in $10^{8} \mathrm{~cm} \cdot \mathrm{s}^{-1}$.

The main results of a parametric exploration of Equation 14 are depicted in Figure 4. In this figure there is shown the synthesized mass of elements between silicon to calcium as a function of the values adopted by the set $\left\{\mathrm{V}_{8}, \mathrm{D}, \tau_{c}\right\}$. In computing Figure 4 we have taken a fixed mass of ashes $M_{N S E E}\left(M_{\odot}\right)=$ 0.8 and $\bar{\rho}_{7}=7$, a density which is slightly higher than the starting density during the exponential expansion. Such combination of $\mathrm{M}_{N S E E}$ and $\bar{\rho}_{7}$ leads to an average radius of the ashes $\bar{r}=1.8 \times 10^{8} \mathrm{~cm}$, in fair agreement to detailed hydrodynamical calculations. For instance, model W7 (Nomoto et al. 1984) gives approximately $r_{8} \simeq 2$ at $\rho_{7}=4$ similar as the one adopted in this work. Going to multidimensional models Röpke et al. 
(2006) give an average radius of the burnt zone also close to $\bar{r}_{8}=2$ in their model c3_3d.

First of all note that the case $\mathrm{D}=2$ in Figure 4 almost always leads to an underproduction of IME. Thus it becomes clear why those one-dimensional models which assumed a spherical burning front moving at the largest turbulent speed, V, did not succeed in synthesizing large amounts of IME (Niemeyer \& Woosley 1997). Also, there is not a sufficient abundance of IME neither for $V_{8}<0.5$ nor for $\tau_{c} \leq 0.05 \mathrm{~s}$. The mass of these elements smoothly rises as $\mathrm{D}, \mathrm{V}$ or $\tau_{c}$ increases, as expected. According to Equation 14 the most influencial parameter affecting the nucleosynthesis of silicon family is the fractal dimension of the underlying iron core, followed by the propagation velocity and the characteristic expansion time respectively.

There are, however, some limitations which could prevent a large production of IME during the explosion. First, the fractal dimension defining the ashes's surface is thought to be closer to 2.3 than to 2.5 because it is highly probable for turbulence to be of Kolmogorov type. Actually, one can expect that the flame behaves as a multifractal, so the effective fractal dimension would be somehow between 2.33 (characteristic of lower scale-lengths) and $\mathrm{D}=2.5$ (characteristic of large scale-lenghts). On the other hand the local sound speed puts an upper limit to the largest turbulent velocity $\mathrm{V}$. At the densities of interest the sound speed is $\simeq 4-4.5 \times 10^{8} \mathrm{~cm} . \mathrm{s}^{-1}$ thus $\mathrm{V}_{8} \simeq 2$ is almost a half of the sound speed. In that case the induction of a detonation because of the cumulative effect of pressure waves can not be ruled out (Plewa et al. 2005). Maybe more interesting are the constraints related to the dominant combustion regime: flamelet or distributed. Taking $\lambda_{\text {max }}=\bar{r}=1.8 \times 10^{8} \mathrm{~cm}, \lambda_{\text {min }} \simeq 0.1 \mathrm{~cm}$ (approximately the flame thickness at these densities), $v_{\text {flame }} \simeq 5 \times 10^{4} \mathrm{~cm} . \mathrm{s}^{-1}$ and $\mathrm{D}^{\prime}=7 / 3$ in Equation 11 the corresponding turbulent velocity is $\mathrm{V} \simeq 6 \times 10^{7} \mathrm{~cm} . \mathrm{s}^{-1}$. With that choice of parameters turbulent combustion will stay in the flamelet regime as long as the velocity of the largest eddy is lower than $6 \times 10^{7} \mathrm{~cm} . \mathrm{s}^{-1}$. Were this the case an inspection of Figure 4 reveals that, even in the most favourable case with $\tau_{c}=0.3 \mathrm{~s}$, the production of IME hardly arrives to $0.2 \mathrm{M}_{\odot}$. Considering stronger turbulent intensities would move the more plausible region for producing IME just to the poorly known distributive regime.

The influence of the characteristic expansion time $\tau_{c}$ is not as strong as that of $\mathrm{V}_{8}$ owing to the inverse dependence of $\mathrm{X}_{I M E}$ with $\tau_{c}$ (Eq. 9). Moreover, one- dimensional simulations indicate that the characteristic expansion time at the time combustion arrives to layers with $\rho \simeq 5 \times 10^{7} \mathrm{~g} . \mathrm{cm}^{-3}$ are probably not much larger than $0.2 \mathrm{~s}$ (see for example Fig. 3). Even though $\tau_{c}$ should be relatively model independent, owing the strong sensitivity of the degenerate matter against perturbations, its precise value is set by the central density at the moment of the explosion and by the nuclear combustion dynamics itself.

Equation 14 suggests that $\mathrm{M}_{I M E}$ and $\mathrm{M}_{N S E E}$ are not independent quantities, $\mathrm{M}_{I M E} \propto M_{N S E E}^{D / 3}$. On the other hand some mass of Fe-peak elements are also produced simultaneously to silicon during the expansion. Thus, the final quantity of Fe-peak, $M_{F e}$ is slightly higher than $M_{N S E E}$. Assuming that after freezing is $X_{F e} \simeq 1-X_{I M E}$ in the IME layer it is easy to show that the total mass of the iron group is:

$M_{F e}\left(M_{\odot}\right)=M_{N S E E}\left(M_{\odot}\right)+M_{I M E}\left(M_{\odot}\right)\left(\frac{\tau_{c}^{1 / 4}}{0.35}-1\right) \quad 0.05 \leq \tau_{c} \leq 0.3$

A plot of $M_{I M E}$ versus $M_{F e}$ for several characteristic expansion times at constant $\mathrm{D}=7 / 3$ and $\mathrm{V}_{8}=1.5$ is given in Figure 5. As we can see the relationship between $M_{I M E}$ and $M_{F e}$ is close to linear with increasing slope as $\tau_{c}$ rises. As a general rule we expect that deflagrations with large luminosities (i.e a large mass of ${ }^{56} \mathrm{Ni}$ ) will also show a large production of IME. Nevertheless some attention has to be paid to the influence of the characteristic expansion time. For example, according to Figure $5,0.2 \mathrm{M}_{\odot}$ of IME can be synthesized in combination to $0.7 \mathrm{M}_{\odot}$ of Fe-peak elements at $\tau_{c}=$ $0.3 \mathrm{~s}$ or to $0.95 \mathrm{M}_{\odot}$ at $\tau_{c}=0.1 \mathrm{~s}$. Hence explosions with different maximum luminosities and global energetics could lead to similar amounts of IME. Moreover, it is reasonable to think that $\tau_{c}$ and $\mathrm{M}_{N S E E}$ are inversely correlated: a large amount of $\mathrm{M}_{N S E E}$ means a big deal of released nuclear energy thus leading to shorter characteristic expansion times. Such correlation suggest that some self-regulating mechanism could be at work reducing the dispersion in the production of IME.

Finally, note that our assumption that ashes are enterily composed of NSEE has not to be necessarily true. Even though there is a current debate concerning this point, recent multidimensional simulations indicate that some unburnt carbon and oxygen could remain mixed with the incinerated material, (García-Senz \& Bravo 2005; Röpke et al. 2006). In that case the correlation between the mass of IME and 
Fe-peak elements will be different to that shown in Figure 5.

\section{Conclusions}

The amount of intermediate-mass elements ejected in a SNIa explosion is an important diagnosing tool to discriminate between a variety of theoretical models attempting to explain the nature of such explosions. One of the most popular models is the deflagration model, rooted in the subsonic propagation of a nuclear flame through the white dwarf interior. However, with very few exceptions, neither the one-dimensional simulations nor modern three-dimensional calculations of this model have been able to give the right quantity of IME. Hydrodynamical calculations always stubbornly give a too low production. This fact is currently attributed to the low resolution achieved in multidimensional calculations.

In the first part of this paper we have analyzed the synthesis of elements from helium to zinc during the propagation of a nuclear flame in an expanding background. The simulation was carried out in the planar aproximation, thus the resolution was enough to resolve the detailed structure of the flame. In this sense our work represents a continuation of that by Timmes $\&$ Woosley (1992). As a result we were able to get an idea of the size of the region where the IME were for the most part synthesized: between $0.15 \mathrm{~km}$ to $0.75 \mathrm{~km}$ depending on the characteristic expansion time. This width is hardly reachable by today multidimensional hydrocodes. It was also possible to give the amount of elements from silicon to calcium cooked behind the flame (Table 1). This information can be used by SNIa modelers to improve the nucleosynthesis output of their hydrodynamical simulations. An interesting result is that IME are massively produced in the range $\rho=2 \times 10^{7}-5 \times 10^{7} \mathrm{~g} . \mathrm{cm}^{-3}$. In fact, according to our calculations $80 \%$ of the IME were already produced before the density declined below $3 \times 10^{7} \mathrm{~g} . \mathrm{cm}^{-3}$ (see Fig. 2). This could have important consequences because the usefulness of our results strongly relies in the basic hypothesis that the flame does not enter the distributive regime above $2-3 \times 10^{7}$ g. $\mathrm{cm}^{-3}$. Finally useful fitting formulae giving the mass of IME synthesized during the expansion as well as to their mass fraction, $\mathrm{X}_{I M E}$, as a function of the characteristic expansion time $\tau_{c}$ were also given (Eqs. 8,9).

In $\S 3$ we did use the information given in Table 1 to estimate the quantity of IME ejected during the deflagration of a white dwarf taking advantage of the self-similar properties of the thermonuclear combustion in SNIa. We have taken a geometrical approach to address this problem in which the narrow region where the Si-peak nuclei are typically produced was treated as a tiny shell wrapping around a deformed surface on top of a volume made of Fe-peak elements. That approach holds as long as the ashes form a well defined and not too dispersed pack limited by an irregular surface. Within our simple model the total amount of elements from silicon to calcium can be obtained from an analytical expression (Eq. 14) which uses four parameters: the ratio between the mass and average density of the NSE-ashes, the fractal dimension of their surface, the velocity of the turbulent combustion at the largest scales, and the characteristic expansion time. In consequence we have tried to delimitate the regions in parameter space able to give the amount of IME compatible with observations (Fig. 4).

As a general conclusion we state the difficulty of pure deflagration models to produce more than $0.2 \mathrm{M}_{\odot}$ of intermediate-mass elements. In this sense our phenomenological approach may help to understand why the underproduction of Si-peak elements has traditionally been a long standing problem of all sort of hydrodynamical simulations concerning Type Ia supernovae assuming the deflagrative paradigma. Still there remains a significant area in space parameter showing a rich production of IME. In particular the region defined by the unequalities $\tau_{c} \geq 0.2 \mathrm{~s}$, $D \geq 2.2$ and $V_{8} \geq 1$ seems to be well suited for that purpose. The above lower limit in the fractal dimension of the ahes's surface does not pose a problem as experiments dealing with turbulent combustion indicates a favoured value of $\mathrm{D}$ close to 2.3. As an aside, 1D simulations that always assume $D=2.0$ on the resolved length-scales lead unavoidably to an underproduction of IME. Perhaps more demanding is the limitation imposed by the characteristic expansion time. Even though $\tau_{c}$ should not be too different from the hydrodynamical time scale, $\simeq 0.1 \mathrm{~s}$ at the densities of interest, its particular value is model dependent. Hence expansion times with $\tau_{c} \simeq 0.2 \mathrm{~s}$ are probably not unrealistic. Turbulent intensities characterized by $V_{8} \geq 1$ are also reachable during the explosion because the rising velocity of the larger floating blobs of incinerated material is of the order, or even greater, than that velocity. Nevertheless, the turbulent cascade generated by these fast moving blobs could fragment the flame in very small pieces, shorter than the flame 
thickness, invalidating our hypothesis that combustion always takes place in the flamelet regime. Thus, although our main goal was to highlight the favoured regions in parameter space to synthesize a good deal of IME, the presented results also underline the potential importance of the distributed regime for deflagration models, if they were to represent the main channel to normal Type Ia supernovae.

Even if a large mass of IME were attained in deflagration models of SNIa, our study still suggests another potential problem: the production of IME seems to increase monothonically with that of Fepeak elements. Hence, it would be seemingly difficult for deflagrations to explain peculiar low-luminosity but energetic events like SN1991bg or SN1998de (Stritzinger et al. 2006).

This work was funded by Spanish DGICYT grant AYA2002-04094 and also supported by DURSI of the Generalitat de Catalunya. SEW acknowledges support from NASA (NNG05GG08G), and the DOE Program for Scientific Discovery through Advanced Computing (SciDAC; DE-FC02-01ER41176). 


\section{REFERENCES}

Bravo, E., Tornambé, A., Dominguez, I., \& Isern, J. 1996, A\&A, 306, 811.

Cabezón, R.M., García-Senz, D., \& Bravo, E. 2005, ApJS, 151, 345.

García-Senz, D. \& Bravo, E. 2005, A\&A, 430, 585

Iwamoto, K., Brachwitz, F., Nomoto, K., Kishimoto, N., Umeda, H., et al. 1999, ApJS, 125, 439.

Khokhlov, A.M. 1995, ApJ, 449, 695.

Khokhlov, A.M., Oran, E.S., \& Wheeler, J.C. 1997, ApJ, 478, 678

Niemeyer, J.,\& Woosley, S.E. 1997, ApJ, 475, 740.

Niemeyer, J., Bushe, W.,K., \& Ruetsch, G.R. 1999, ApJ, 524, 290.

Nomoto, K., Thielemann, F.-K., \& Yokoi, K. 1984, ApJ, 286, 644.

Peters, N.,\& Franke, Ch. 1989, in Dissipative Structures, Transport Processes \& Combustion, ed. D. meinkön (Berlin: Springer), 40.

Plewa, T., Calder, A.C., \& Lamb, D.Q. 2004, ApJ, 612, L37.

Röpke, F.K., Hillebrandt, W., Niemeyer, J.C., \& Woosley, S.E. 2006, A\&A, 448, 1.

Stritzinger, M., Leibundgut, B., Walch, S., \& Contardo, G. 2006, A\&A, 450, 241

Timmes, F.X.,\& Woosley, S.E. 1992, ApJ, 396, 649.

Woosley, S.E. 2001, Nucl. Phys., A688, 9c.

Zingale, M., Woosley, S.E., Rendleman, C.A., Day, M.S., \& Bell, J.B. 2005, ApJ, 632, 1021.

This 2-column preprint was prepared with the AAS ${ }^{\mathrm{A}} \mathrm{T}_{\mathrm{E}} \mathrm{X}$ macros v5.2. 


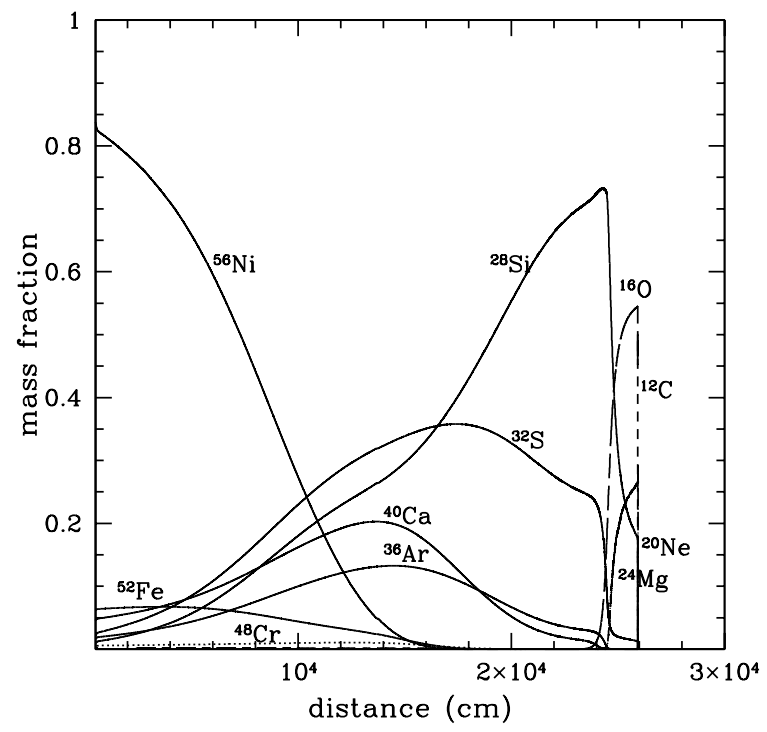

Fig. 1.- Mass fraction profile of the most abundant species synthesized during the decay of a planar nuclear flame due to expansion. A characteristic expansion time $\tau_{c}=0.1 \mathrm{~s}$ was assumed in this case. The elapsed time was $\mathrm{t}=0.1523 \mathrm{~s}$, which corresponds to a fuel density of $1.45 \times 10^{7} \mathrm{~g} . \mathrm{cm}^{-3}$. Below that density the total amount of IME practically freezes 


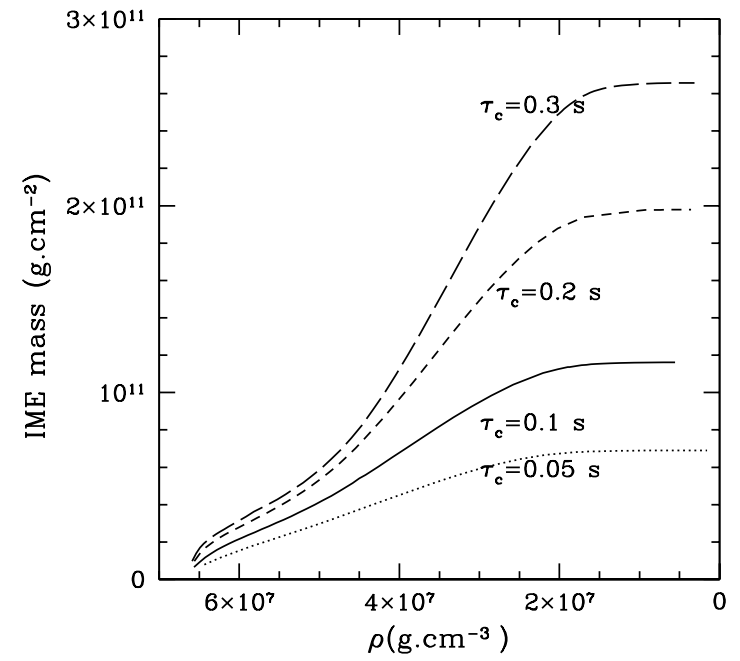

Fig. 2.- Total mass per unit area of IME (from silicon to calcium) synthesized during the isobaric propagation of the flame as a function of the instantaneous density value in the cold fuel for four different characteristic expansion times. At $\rho=2 \times 10^{7} \mathrm{~g} . \mathrm{cm}^{-3}$ practically all IME have already been produced. 


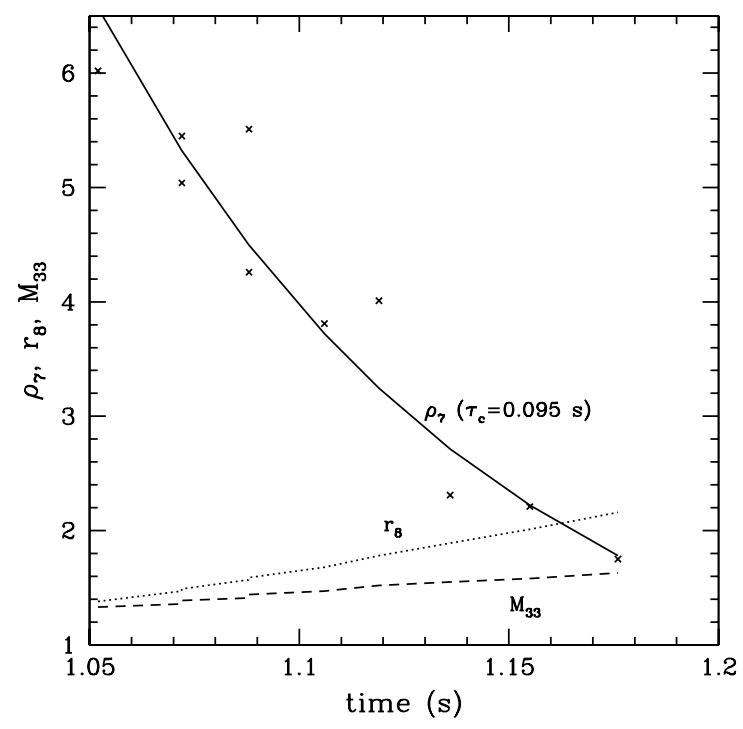

Fig. 3.- Evolution of density, radius and lagrangianmass coordinate (in $10^{7}, 10^{8}$ and $10^{33}$ c.g.s units respectively) of the one-dimensional model used to compute some of the inputs of Figure 4. The figure depicts the value of these variables just at the moment the temperature of the corresponding mass-shell becomes higher than one billion degrees. Thus, crosses represent the density of different mass-shells as they are reached by the flame. The solid line is an exponential fit to the numerical data using a characteristic time $\tau_{c}=0.095 \mathrm{~s}$. We have checked that the maximum temperature achieved by these shells is in the right range to give intermediate-mass elements. 

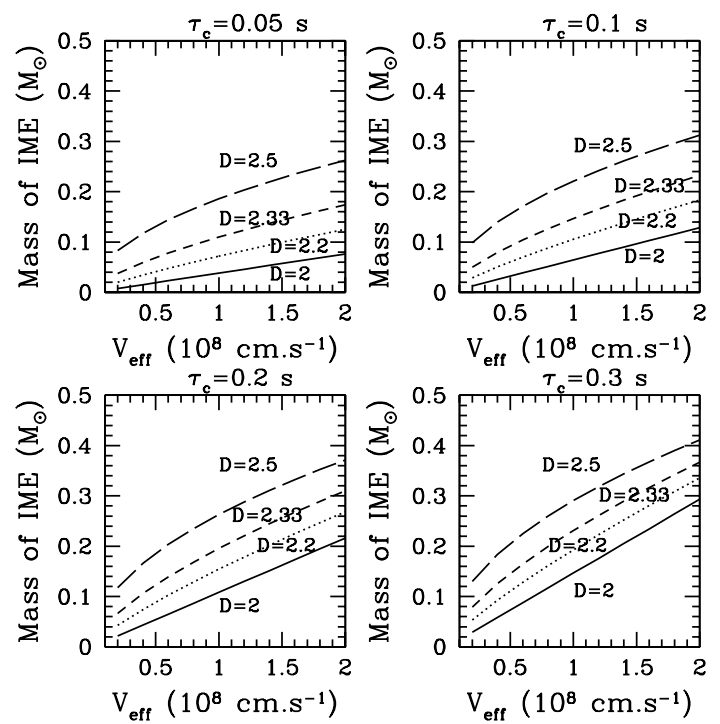

Fig. 4.- Total amount (in $\mathrm{M}_{\odot}$ ) of elements from silicon to calcium synthesized during the expansion of a massive white dwarf due to the propagation of a turbulent nuclear flame. The region where these nuclei are produced is approximated by a fractal of dimension $\mathrm{D}$ and average radius $\bar{r}=1.8 \times 10^{8} \mathrm{~cm}$. The quantity of IME is then given as a function of $\mathrm{D}$, the effective propagation velocity of the flame $V_{\mathrm{e} f f}$, and the characteristic expansion time $\tau_{c}$. 


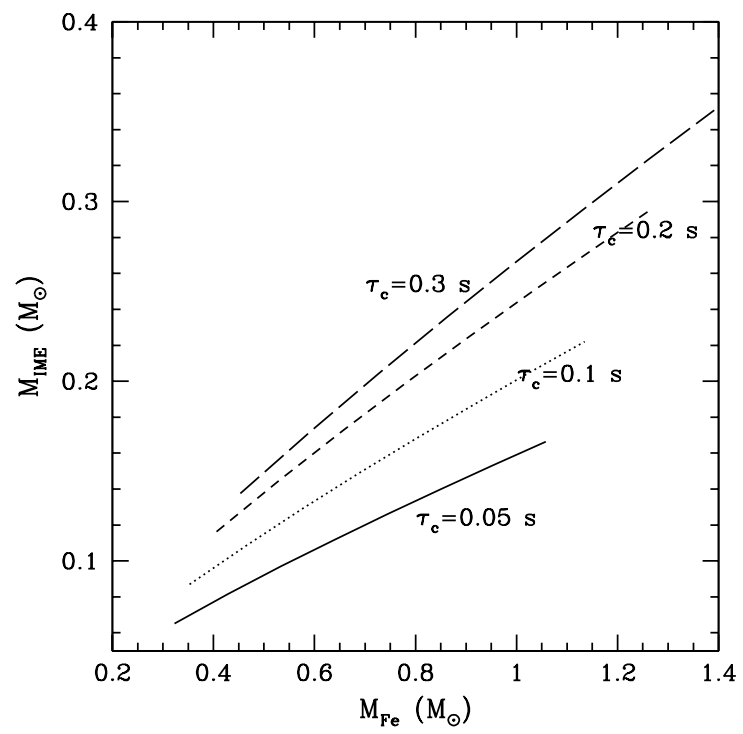

Fig. 5.- Mass of IME as a function of the ejected mass of Fe-peak elements for several expansion times. A constant fractal dimension of the iron core surface, $\mathrm{D}=7 / 3$, was assumed as well as a constant turbulence intensity, $\mathrm{V}=1.5 \times 10^{8} \mathrm{~cm} \cdot \mathrm{s}^{-1}$. 
Table 1: Final masses $\left(\mathrm{g} . \mathrm{cm}^{-2}\right)$ of $\alpha$-elements produced in a laminar expanding nuclear flame for several characteristic expansion times

\begin{tabular}{crrrr}
\hline Nuclei & $\tau_{c}=0.05 \mathrm{~s}$ & $\begin{array}{r}\tau_{c}=0.1 \mathrm{~s} \\
\text {-mass }\left(\mathrm{g} . \mathrm{cm}^{-2}\right)-\end{array}$ & $\tau_{c}=0.2 \mathrm{~s}$ & $\tau_{c}=0.3 \mathrm{~s}$ \\
\hline${ }^{4} \mathrm{He}$ & $4.110^{-2}$ & $1.6210^{-1}$ & $4.2510^{5}$ & $2.710^{7}$ \\
${ }^{12} \mathrm{C}$ & $3.310^{8}$ & $3.4810^{8}$ & $3.7210^{8}$ & $3.910^{8}$ \\
${ }^{16} \mathrm{O}$ & $4.910^{9}$ & $7.710^{9}$ & $1.2310^{10}$ & $1.610^{10}$ \\
${ }^{20} \mathrm{Ne}$ & $3.510^{7}$ & $4.410^{7}$ & $5.6010^{7}$ & $6.910^{7}$ \\
${ }^{24} \mathrm{Mg}$ & $1.910^{9}$ & $3.210^{9}$ & $5.1210^{9}$ & $6.910^{9}$ \\
${ }^{28} \mathrm{Si}$ & $2.9510^{10}$ & $4.9810^{10}$ & $8.5710^{10}$ & $1.1610^{11}$ \\
${ }^{32} \mathrm{~S}$ & $2.210^{10}$ & $3.6910^{10}$ & $6.2510^{10}$ & $8.410^{10}$ \\
${ }^{36} \mathrm{Ar}$ & $7.310^{9}$ & $1.2110^{10}$ & $2.010^{10}$ & $2.610^{10}$ \\
${ }^{40} \mathrm{Ca}$ & $1.010^{10}$ & $1.7510^{10}$ & $2.9710^{10}$ & $3.910^{10}$ \\
${ }^{44} \mathrm{Ti}$ & $1.310^{7}$ & $2.0210^{7}$ & $1.010^{8}$ & $5.210^{8}$ \\
${ }^{48} \mathrm{Cr}$ & $5.610^{8}$ & $9.710^{8}$ & $1.6510^{9}$ & $2.210^{9}$ \\
${ }^{52} \mathrm{Fe}$ & $2.310^{9}$ & $5.310^{9}$ & $1.1510^{10}$ & $1.710^{10}$ \\
${ }^{56} \mathrm{Ni}$ & $1.4610^{10}$ & $4.610^{10}$ & $1.4010^{11}$ & $2.610^{11}$ \\
${ }^{60} \mathrm{Zn}$ & $1.010^{3}$ & $2.710^{3}$ & $3.2710^{5}$ & $1.510^{7}$ \\
\hline
\end{tabular}


Table 2: Estimated amounts of IME synthesized during an exponential expansion from $\rho_{0}=6.6 \times 10^{7} \mathrm{~g} . \mathrm{cm}^{-3}$

down to $\rho_{0}=2 \times 10^{6}$ g.cm ${ }^{-3}$ with characteristic time $\tau_{c}$

\begin{tabular}{ccrrrr}
\hline $\begin{array}{c}\tau_{c} \\
(\mathrm{~s})\end{array}$ & $\begin{array}{r}\Delta m_{\text {burnt }} \\
\mathrm{g} . \mathrm{cm}^{-2}\end{array}$ & $\begin{array}{r}\Delta m_{I M E} \\
\mathrm{~g} . \mathrm{cm}^{-2}\end{array}$ & $\begin{array}{r}\Delta m_{N i} \\
\mathrm{~g} . \mathrm{cm}^{-2}\end{array}$ & $\begin{array}{r}\mathrm{X}_{I M E} \\
-\end{array}$ & $\begin{array}{r}\mathrm{X}_{N i} \\
-\end{array}$ \\
\hline 0.05 & $9.2710^{10}$ & $6.8810^{10}$ & $1.7510^{10}$ & 0.742 & 0.188 \\
0.1 & $1.8010^{11}$ & $1.1610^{11}$ & $5.2310^{10}$ & 0.646 & 0.29 \\
0.2 & $3.6910^{11}$ & $1.9810^{11}$ & $1.5310^{11}$ & 0.537 & 0.415 \\
0.3 & $5.6810^{11}$ & $2.6510^{11}$ & $2.810^{11}$ & 0.466 & 0.492 \\
\hline
\end{tabular}

